\title{
Synthesis of 2-trifluoromethylpyrazolo[5,1-a]isoquinolines via silver triflate-catalyzed or electrophile-mediated one-pot tandem reaction
}

\author{
Xiaoli Zhou ${ }^{1}$, Meiling Liu ${ }^{1}$, Puying Luo ${ }^{*}$, Yingjun Lai ${ }^{1}$, Tangtao Yang ${ }^{1}$ \\ and Qiuping Ding ${ }^{*} 1, \S$
}

\author{
Full Research Paper \\ Address: \\ ${ }^{1}$ Key Laboratory of Small Functional Organic Molecule, Ministry of \\ Education and Jiangxi's Key Laboratory of green chemistry, Jiangxi \\ Normal University, Nanchang, Jiangxi 330022, P. R. China and \\ ${ }^{2}$ Department of Obstetrics and Gynecology, Jiangxi Provincial \\ people's Hospital, Nanchang, Jiangxi 330006, P. R. China \\ Email: \\ Puying Luo* - luopuying1979@gmail.com; Quuping Ding* - \\ dingqiuping@jxnu.edu.cn \\ * Corresponding author \\ § Tel.: +86 79188120380 ; fax: +86 79188120380 \\ Keywords: \\ [3 + 2] cycloaddition; electrophile; $N^{\prime}$-(2-alkynylbenzylidene)hydrazide; \\ silver triflate; tandem
}

Beilstein J. Org. Chem. 2014, 10, 2286-2292.

doi:10.3762/bjoc. 10.238

Received: 19 May 2014

Accepted: 18 September 2014

Published: 30 September 2014

Associate Editor: D. O'Hagan

(C) 2014 Zhou et al; licensee Beilstein-Institut. License and terms: see end of document.

\begin{abstract}
An efficient one-pot tandem cyclization/[3 + 2] cycloaddition reaction of $N^{\prime}$-(2-alkynylbenzylidene)hydrazides with ethyl 4,4,4trifluorobut-2-ynoate under silver triflate-catalyzed or electrophile-mediated conditions is described. Various trifluoromethylated pyrazolo[5,1-a]isoquinolines were afforded in moderate to excellent yield by this developed method.
\end{abstract}

\section{Introduction}

Isoquinolines and isoquinoline-derived heterocycles are prevalent structural motifs in natural products and pharmaceuticals that exhibit remarkable biological activities $[1,2]$. Therefore, great attention has been directed toward the development of efficient methods for the selective functionalization of the isoquinoline cores. Among these, pyrazolo[5,1-a]isoquinoline is an important class of isoquinoline derivatives. Recently, much effort has been spent on the synthesis of these compounds due to their promising biological activities [3-18]. For instance, in 2010, Wu and co-workers found some pyrazolo[5,1- $a$ isoquino- line derivatives showing activities for the inhibition of CDC25B, TC-PTP, and PTP1B [4].

It has been proved that the physical, chemical, and biological activity of organic molecules can be dramatically improved by substitution of hydrogen with fluorine atoms because of the strong electronegativity, the small size, the strength of the $\mathrm{C}-\mathrm{F}$ bond, and the low polarizability of the fluorine atom. Statistically, more than $20 \%$ of the pharmaceuticals and $40 \%$ of the agrochemicals contain one or more fluorine atoms. Thus, there 
has been considerable interest in developing an efficient method for the synthesis of fluorinated heterocycles. Perfluoroalkynoate is a versatile and powerful building block for generating functionalized perfluoroalkylated compounds, especially fluorinated heterocycles, by tandem reactions [19-26]. For example, 2-perfluoroalkynoates have been widely used in synthesizing fluorinated heterocycles, such as benzodiazepines [22], chromenes [21,25], and 2-oxopyridine-fused 1,3-diazaheterocycles [26].

As part of our ongoing efforts in developing synthetic approaches for the functionalization of isoquinoline cores $[11,14]$ and the synthesis of novel fluorinated heterocycles [27] with potential biological applications, herein, we describe an efficient method for the one-pot synthesis of trifluoromethylated pyrazolo[ $[5,1-a]$ isoquinoline derivates via a Lewis acid (AgOTf) or an electrophile- ( $\mathrm{I}_{2}$ or $\mathrm{ICl}$ ) promoted annulation of $N^{\prime}$-(2-alkynylbenzylidene)hydrazides followed by an 1,3-dipolar cycloaddition.

\section{Results and Discussion}

Based on Wu's work on the silver triflate-catalyzed tandem reaction of $N^{\prime}$-(2-alkynylbenzylidene)hydrazides with dimethyl acetylenedicarboxylate [28], we started our research by examining the reaction of $N^{\prime}$-(2-alkynylbenzylidene)hydrazides $\mathbf{1 a}$ (0.3 mmol) and ethyl 4,4,4-trifluorobut-2-ynoate (2, $0.6 \mathrm{mmol})$ using NaOAc $(0.45 \mathrm{mmol})$ as base, in the presence of AgOTf (5 mol \%) and $4 \AA \mathrm{MS}(75 \mathrm{mg})$ in $\mathrm{CH}_{2} \mathrm{Cl}_{2}(3 \mathrm{~mL})$ at room temperature overnight. Surprisingly, the unexpected $[3+2]$ cycloaddition product pyrazolo[5,1-a] isoquinoline derivative 3a instead of the isoquinoline-based azomethine ylide [28] was obtained in good yield ( $84 \%$, Table 1 , entry 1$)$. Similar yields were obtained when $\mathrm{NaHCO}_{3}$ or $\mathrm{K}_{2} \mathrm{CO}_{3}$ were used as base ( $83 \%$ and $86 \%$ yield, respectively, Table 1, entries 2 and 3 ). Several other inorganic or organic bases were examined, and the results showed that $\mathrm{CsF}$ was the best choice $(91 \%$ yield, Table 1, entry 8). The control experiment also showed that the base was important for the reaction to proceed ( $8 \%$ yield, Table 1, entry 13). Subsequently, a range of solvents, such as

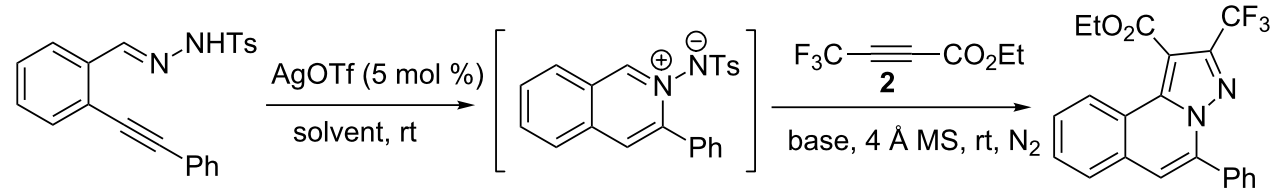

$1 \mathbf{a}$
$3 \mathbf{a}$

\begin{tabular}{|c|c|c|c|}
\hline Entry & Base (1.5 equiv) & Solvent & Yield $(\%)^{b}$ \\
\hline 1 & $\mathrm{NaOAc}$ & $\mathrm{CH}_{2} \mathrm{Cl}_{2}$ & 84 \\
\hline 2 & $\mathrm{NaHCO}_{3}$ & $\mathrm{CH}_{2} \mathrm{Cl}_{2}$ & 83 \\
\hline 3 & $\mathrm{~K}_{2} \mathrm{CO}_{3}$ & $\mathrm{CH}_{2} \mathrm{Cl}_{2}$ & 86 \\
\hline 4 & $\mathrm{Cs}_{2} \mathrm{CO}_{3}$ & $\mathrm{CH}_{2} \mathrm{Cl}_{2}$ & 63 \\
\hline 5 & $\mathrm{NaOH}$ & $\mathrm{CH}_{2} \mathrm{Cl}_{2}$ & 51 \\
\hline 6 & $t$-BuOK & $\mathrm{CH}_{2} \mathrm{Cl}_{2}$ & trace \\
\hline 7 & $\mathrm{KF}$ & $\mathrm{CH}_{2} \mathrm{Cl}_{2}$ & 80 \\
\hline 8 & $\mathrm{CsF}$ & $\mathrm{CH}_{2} \mathrm{Cl}_{2}$ & 91 \\
\hline 9 & DABCO & $\mathrm{CH}_{2} \mathrm{Cl}_{2}$ & 60 \\
\hline 10 & $\mathrm{NEt}_{3}$ & $\mathrm{CH}_{2} \mathrm{Cl}_{2}$ & 61 \\
\hline 11 & DBU & $\mathrm{CH}_{2} \mathrm{Cl}_{2}$ & 50 \\
\hline 12 & Pyridine & $\mathrm{CH}_{2} \mathrm{Cl}_{2}$ & 37 \\
\hline 13 & - & $\mathrm{CH}_{2} \mathrm{Cl}_{2}$ & 8 \\
\hline 14 & $\mathrm{CsF}$ & $\mathrm{CH}_{3} \mathrm{CN}$ & 65 \\
\hline 15 & $\mathrm{CsF}$ & toluene & 69 \\
\hline 16 & $\mathrm{CsF}$ & THF & 71 \\
\hline 17 & $\mathrm{CsF}$ & dioxane & 76 \\
\hline 18 & $\mathrm{CsF}$ & DMA & 87 \\
\hline 19 & CsF (1.0 equiv) & $\mathrm{CH}_{2} \mathrm{Cl}_{2}$ & 65 \\
\hline
\end{tabular}

aReaction conditions: $N^{\prime}$-(2-alkynylbenzylidene)hydrazide 1a (0.3 mmol), AgOTf (5 mol \%), solvent (3 mL), ethyl 4,4,4-trifluorobut-2-ynoate (2, $0.6 \mathrm{mmol}, 2.0$ equiv), base (1.5 equiv), $4 \AA \mathrm{MS}(75 \mathrm{mg})$, rt, overnight. ${ }^{\mathrm{b}}$ Isolated yield based on $1 \mathrm{a}$. 
acetonitrile, toluene, THF, dioxane and DMA were screened, and the results revealed that $\mathrm{CH}_{2} \mathrm{Cl}_{2}$ was the best one, and most of the others were exhibited good yields (Table 1, entry 8 and entries 14-18). The yield (65\%) was reduced obviously when the loading of base was decreased to 1.0 equiv (Table 1 , entry 19).
To explore the scope of this tandem cyclization/[3+2] cycloaddition reaction, a range of $N^{\prime}$-(2-alkynylbenzylidene)hydrazides $\mathbf{1 a}-\mathbf{j}$ were prepared from the corresponding aldehydes and applied to the synthesis of trifluoromethylated pyrazolo[5,1a] isoquinoline derivatives 3 under the optimized conditions (Table 1, entry 8). As shown in Table 2, for most cases, $N^{\prime}-(2-$

Table 2: Silver triflate-catalyzed tandem reactions of $N^{\prime}$-(2-alkynylbenzylidene)hydrazides 1 with ethyl 4,4,4-trifluorobut-2-ynoate 2.
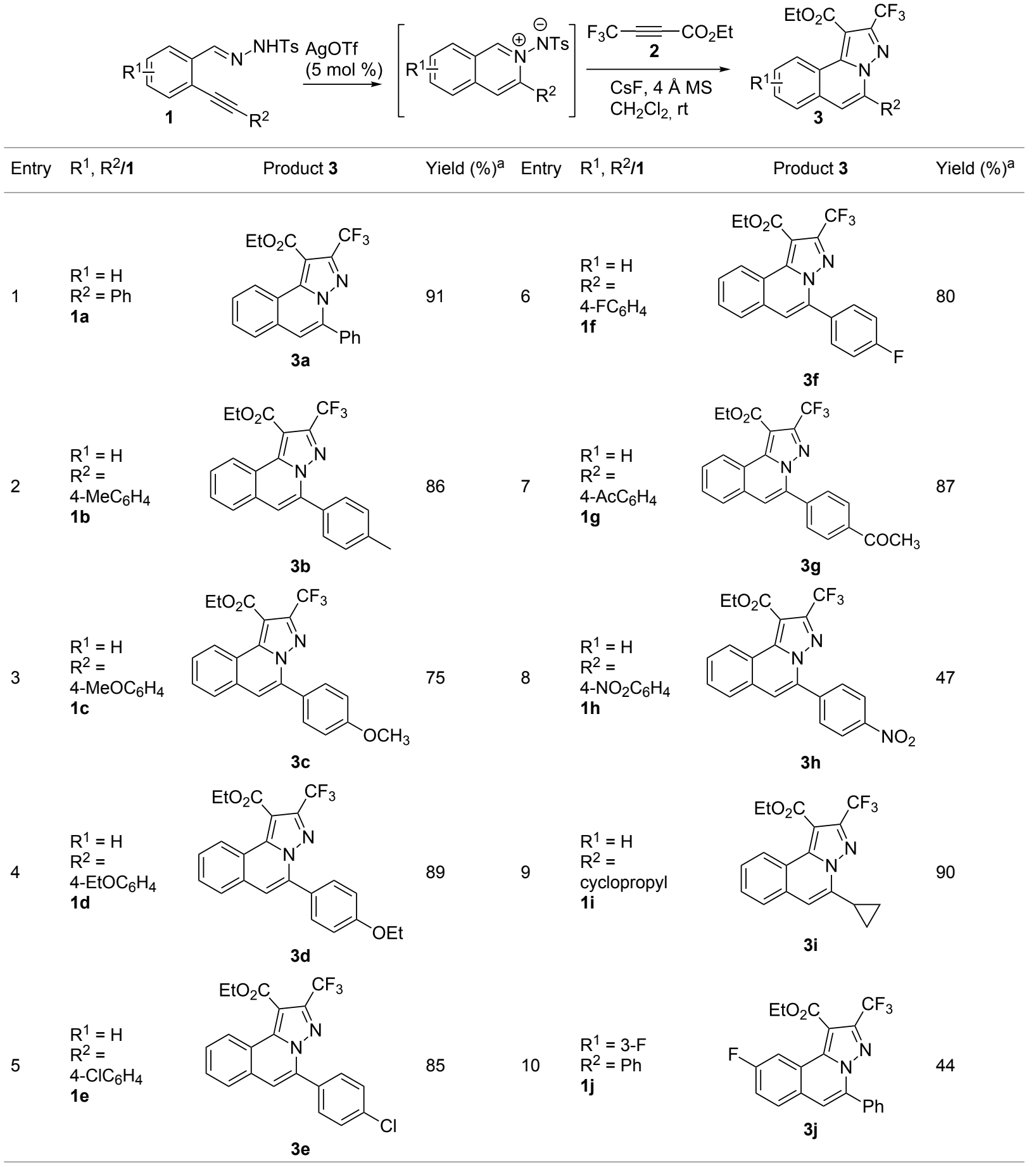

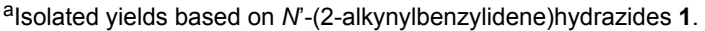


alkynylbenzylidene)hydrazides 1 reacted with ethyl 4,4,4-trifluorobut-2-ynoate $\mathbf{2}$ affording the corresponding products $\mathbf{3}$ in good to excellent yields. For instance, substrate $\mathbf{1 b}$ bearing an electron-donating substituent (methyl) reacted with 2 under the present reaction conditions gave the desired product $\mathbf{3 b}$ in good yield ( $86 \%$, Table 2 , entry 2 ). The structure of $\mathbf{3 b}$ was verified by ${ }^{1} \mathrm{H}$ and ${ }^{13} \mathrm{C}$ NMR, HRMS, as well as X-ray diffraction analysis (Figure 1, for details, see Supporting Information File 1). As expected, the substrates $\mathbf{1 e - h}$ with electron-withdrawing substituents are suitable partners in this process and the corresponding pyrazolo[5,1- $a$ ] isoquinolines $\mathbf{3 e}-\mathbf{h}$ were obtained in good yields. Fortunately, alkyl-substituted $N^{\prime}$-(2-alkynylbenzylidene)hydrazide was demonstrated to be good partner in the transformation. For instance, N'-(2-alkynylbenzylidene)hydrazide $1 \mathbf{i}$ reacted with 2 , leading to the desired pyrazolo[5,1a]isoquinoline $3 \mathbf{i}$ in $90 \%$ yield (Table 2 , entry 9 ).

Subsequently, based on our previous reports on electrophilemediated electrophilic cyclization reaction [28,29], one-pot tandem electrophilic cyclization/[3 + 2] cycloaddition of $N^{\prime}-(2-$ alkynylbenzylidene)hydrazides $\mathbf{1}$, electrophiles ( $\mathrm{I}_{2}$ or $\mathrm{ICl}$ ), and ethyl 4,4,4-trifluorobut-2-ynoate (2) were carried out under mild conditions. The results are summarized in Table 3. For all cases, this tandem reaction worked well leading to the corresponding iodinated fluorine-containing pyrazolo[5,1-a]isoquinolines $\mathbf{4}$ in moderate to excellent yields. Various functional groups, such as methyl, methoxy, ethoxy, halogen, acetyl, nitro, and cyclopropyl groups were tolerated under the reaction conditions. In general, substrates bearing electron-donating substituents show better reactivity than those with electronwithdrawing substituents. For instance, methyl-substituted $N^{\prime}$-(2-alkynylbenzylidene)hydrazide $\mathbf{1 b}$ reacted with iodine and ethyl 4,4,4-trifluorobut-2-ynoate (2) in the presence of CsF and $4 \AA$ MS leading to the desired product $4 \mathbf{b}$ in $90 \%$ yield (Table 3, entry 3). A relatively lower yield was obtained when nitro substituted $N^{\prime}$-(2-alkynylbenzylidene)hydrazide $\mathbf{1 h}$ was used, and the desired product $\mathbf{4 g}$ was obtained in $50 \%$ yield (Table 3, entry 9). Alkyl-substituted product $\mathbf{4 h}$ was obtained in good yields when substrate 1 i reacted with iodine or ICl (Table 3, entries 10 and 11), the structure of $\mathbf{4 h}$ was verified by
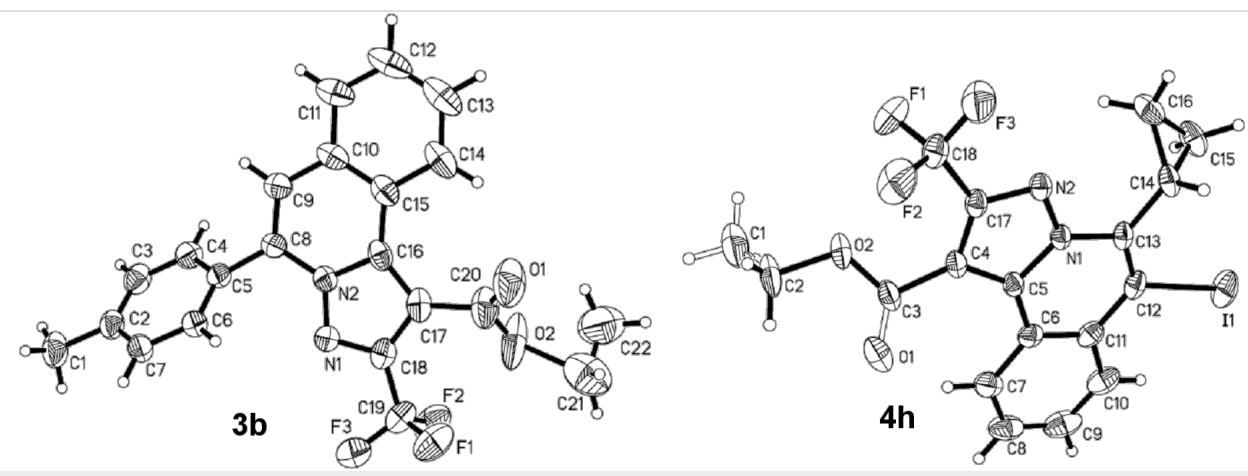

Figure 1: ORTEP diagrams of $3 \mathrm{~b}$ and $\mathbf{4 h}$.

Table 3: One-pot tandem reactions of $N^{\prime}$-(2-alkynylbenzylidene)hydrazides 1, electrophiles, and ethyl 4,4,4-trifluorobut-2-ynoate (2) ${ }^{\text {a }}$.<smiles>[R]C#CC1=CC=C[R1]C=C1/C=N\N[Sb]</smiles>

\begin{tabular}{|c|c|c|c|c|c|c|c|c|c|}
\hline Entry & 1 & $x_{2}$ & 4 & Yield $(\%)^{b}$ & Entry & 1 & $x_{2}$ & 4 & Yield $(\%)^{b}$ \\
\hline 1 & $1 a$ & $\mathrm{I}_{2}$ & $4 a$ & 71 & 7 & $1 f$ & $\mathrm{I}_{2}$ & & 62 \\
\hline
\end{tabular}


Table 3: One-pot tandem reactions of $N^{\prime}$-(2-alkynylbenzylidene)hydrazides 1, electrophiles, and ethyl 4,4,4-trifluorobut-2-ynoate (2) ${ }^{\mathrm{a}}$. (continued)

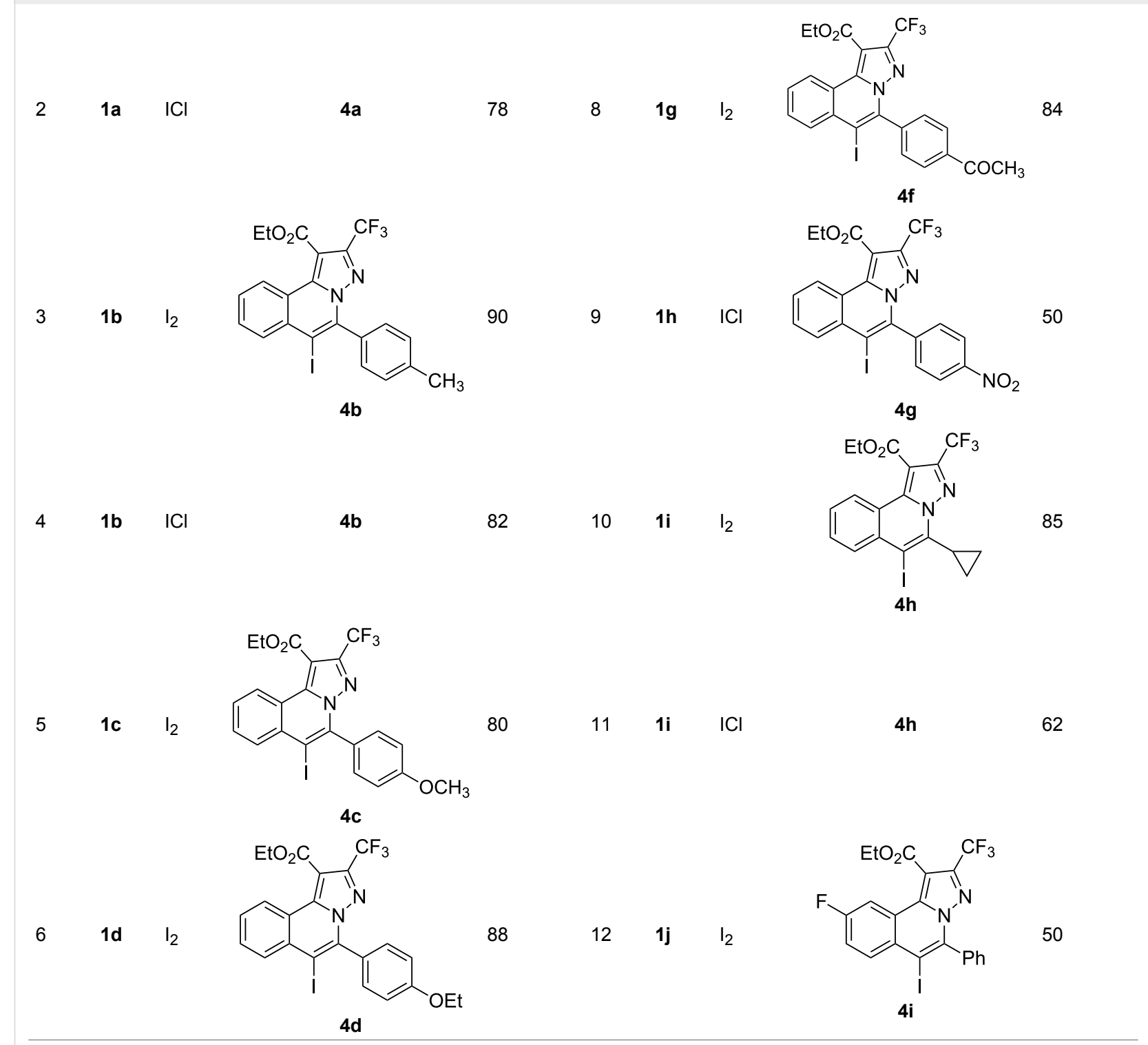

aReaction conditions: $N^{\prime}$-(2-alkynylbenzylidene)hydrazide 1 a $(0.3 \mathrm{mmol}), \mathrm{I}_{2}$ or ICl (1.3 equiv), solvent (3 mL), ethyl 4,4,4-trifluorobut-2-ynoate (2, $0.6 \mathrm{mmol}, 2.0$ equiv), base (1.5 equiv), $4 \AA \mathrm{MS}(75 \mathrm{mg})$, rt, overnight. ' $/$ Isolated yields based on N'-(2-alkynylbenzylidene)hydrazides 1.

${ }^{1} \mathrm{H}$ and ${ }^{13} \mathrm{C}$ NMR, HRMS, as well as X-ray diffraction analysis (Figure 1, for details, see Supporting Information File 1). Based on this one-pot tandem electrophilic cyclization/[3 + 2] cycloaddition reactions, highly functionalized pyrazolo[ $[5,1-a]$ isoquinolines can be obtained via palladium-catalyzed cross-coupling reaction.

\section{Conclusion}

In conclusion, we have developed an efficient one-pot tandem cyclization/[3 + 2] cycloaddition reaction of $N^{\prime}$-(2-alkynylbenzylidene)hydrazides with ethyl 4,4,4-trifluorobut-2-ynoate under silver triflate-catalyzed or electrophiles-mediated conditions. Highly functionalized pyrazolo[ $[5,1-a]$ isoquinolines can be synthesized in moderate to excellent yield by this developed method.

\section{Experimental \\ General}

All reactions were performed in test tubes under $\mathrm{N}_{2}$-atmosphere. Flash column chromatography was performed with silica gel (200-300 mesh). Analytical thin-layer chromatography was performed on glass plates pre-coated with $0.25 \mathrm{~mm}$ 230-400 mesh silica gel impregnated with a fluorescent indicator $(254 \mathrm{~nm})$. Thin-layer chromatography plates were visualized by exposure to ultraviolet light. Organic solutions were concentrated on rotary evaporators at $25-35^{\circ} \mathrm{C}$. Commercial 
reagents and solvents were used as received. ${ }^{1} \mathrm{H}$ and ${ }^{13} \mathrm{C}$ NMR spectra were recorded on a Bruker AV 400 at $400 \mathrm{MHz}\left({ }^{1} \mathrm{H}\right)$ and $100 \mathrm{MHz}\left({ }^{13} \mathrm{C}\right)$ at ambient temperature. Chemical shifts are reported in parts per million (ppm) on the delta scale $(\delta)$ and referenced to tetramethylsilane (0 ppm). HRMS analyses were performed in ESI mode on a Bruker mass spectrometer.

General procedure for the silver triflate-catalyzed one-pot tandem reaction of $N^{\prime}$-(2-alkynylbenzylidene)hydrazide 1 with ethyl 4,4,4-trifluorobut-2-ynoate 2: A mixture of $N^{\prime}$-(2-alkynylbenzylidene)hydrazide 1 ( $0.30 \mathrm{mmol}, 1.0$ equiv) and silver triflate $(5 \mathrm{~mol} \%)$ in anhydrous dichloromethane $(3.0 \mathrm{~mL})$ was stirred at room temperature overnight. Then a solution of ethyl 4,4,4-trifluorobut-2-ynoate ( $2,0.60 \mathrm{mmol}, 2.0$ equiv) in dichloroethane $(2.0 \mathrm{~mL}), 4 \AA \mathrm{MS}(75 \mathrm{mg})$ and $\mathrm{CsF}(0.45 \mathrm{mmol}$, 1.5 equiv) were added and stirred for another $3 \mathrm{~h}$. After completion of the reaction as indicated by TLC, the reaction mixture was purified by flash column chromatography on silica gel to provide the corresponding product $\mathbf{3}$.

General procedure for the electrophile-mediated one-pot tandem reaction of $N^{\prime}$-(2-alkynylbenzylidene)hydrazide 1 with ethyl 4,4,4-trifluorobut-2-ynoate (2): A mixture of $N^{\prime}$-(2-alkynylbenzylidene)hydrazide 1 ( $0.30 \mathrm{mmol}, 1.0$ equiv) and electrophiles ( $\mathrm{I}_{2}$ or $\left.\mathrm{ICl}\right)(0.36 \mathrm{mmol}, 1.2$ equiv) in anhydrous dichloromethane $(3.0 \mathrm{~mL})$ was stirred at room temperature overnight. Then a solution of ethyl 4,4,4-trifluorobut-2-ynoate (2, $0.60 \mathrm{mmol}, 2.0$ equiv) in dichloroethane $(2.0 \mathrm{~mL}), 4 \AA \mathrm{MS}$ ( $75 \mathrm{mg}$ ) and $\mathrm{CsF}$ ( $0.45 \mathrm{mmol}, 1.5$ equiv) were added and stirred for another $3 \mathrm{~h}$. After completion of the reaction as indicated by TLC, the reaction mixture was purified by flash column chromatography on silica gel to provide the corresponding product 4. For details, see Supporting Information File 1.

\section{Supporting Information}

\section{Supporting Information File 1 \\ Characterization data and NMR spectra. \\ [http://www.beilstein-journals.org/bjoc/content/ supplementary/1860-5397-10-238-S1.pdf]}

\section{Supporting Information File 2}

$\mathrm{X}$-ray data for compound $\mathbf{3 b}$.

[http://www.beilstein-journals.org/bjoc/content/ supplementary/1860-5397-10-238-S2.cif]

\section{Supporting Information File 3}

X-ray data for compound $\mathbf{4 h}$.

[http://www.beilstein-journals.org/bjoc/content/ supplementary/1860-5397-10-238-S3.cif]

\section{Acknowledgements}

Financial Supported from the National Natural Science Foundation of China (21262016), Jiangxi Educational Committee (GJJ12169), the Project of Jiangxi Youth Scientist (20122BCB23012), and Natural Science Foundation of Jiangxi Province of China (20133ACB20008, 20132BAB203006) is gratefully acknowledged.

\section{References}

1. Bentley, K. W. The Isoquinoline Alkaloids; Harwood Academic Publishers: Australia, 1998; Vol. 1.

2. Trotter, B. W.; Nanda, K. K.; Kett, N. R.; Regan, C. P.; Lynch, J. J.; Stump, G. L.; Kiss, L.; Wang, J.; Spencer, R. H.; Kane, S. A.; White, R. B.; Zhang, R.; Anderson, K. D.; Liverton, N. J.; Mclntyre, C. J.; Beshore, D. C.; Hartman, G. D.; Dinsmore, C. J. J. Med. Chem. 2006, 49, 6954-6957. doi:10.1021/jm060927v

3. Mousseau, J. J.; Fortier, A.; Charette, A. B. Org. Lett. 2010, 12, 516-519. doi:10.1021/ol902710f

4. Chen, Z.; Wu, J. Org. Lett. 2010, 12, 4856-4859. doi:10.1021/ol101988q

5. Li, X.; Zhao, M. J. Org. Chem. 2011, 76, 8530-8536. doi:10.1021/jo201530r

6. Li, S.; Wu, J. Org. Lett. 2011, 13, 712-715. doi:10.1021/ol102939r

7. Huple, D. B.; Chen, C.-H.; Das, A.; Liu, R.-S. Adv. Synth. Catal. 2011, 353, 1877-1882. doi:10.1002/adsc.201100263

8. Zhao, J.; Li, P.; Wu, C.; Chen, H.; Ai, W.; Sun, R.; Ren, H.; Larock, R. C.; Shi, F. Org. Biomol. Chem. 2012, 10, 1922-1930. doi:10.1039/c2ob06611d

9. Chen, Z.; Gao, L.; Ye, S.; Ding, Q.; Wu, J. Chem. Commun. 2012, 48, 3975-3977. doi:10.1039/c2cc30413a

10. Huang, P.; Yang, Q.; Chen, Z.; Ding, Q.; Xu, J.; Peng, Y. J. Org. Chem. 2012, 77, 8092-8098. doi:10.1021/jo3013429

11. Ding, Q.; Wang, D.; Sang, X.; Lin, Y.; Peng, Y. Tetrahedron 2012, 68, 8869-8874. doi:10.1016/j.tet.2012.08.039

12. Xu, S.-X.; Hao, L.; Wang, T.; Ding, Z.-C.; Zhan, Z.-P. Org. Biomol. Chem. 2013, 11, 294-298. doi:10.1039/c2ob27016a

13. Liu, H.; Liu, G.; Qiu, G.; Pu, S.; Wu, J. Tetrahedron 2013, 69 , 1476-1480. doi:10.1016/j.tet.2012.12.018

14. Ding, Q.; Wang, D.; Luo, P.; Liu, M.; Pu, S.; Zhou, L. Beilstein J. Org. Chem. 2013, 9, 1949-1956. doi:10.3762/bjoc.9.231

15. Xiao, Q.; Zheng, D.; Ding, Q.; Wu, J. Tetrahedron 2013, 69, 5119-5122. doi:10.1016/j.tet.2013.04.076

16. Pan, X.; Luo, Y.; Wu, J. J. Org. Chem. 2013, 78, 5756-5760. doi:10.1021/jo400523v

17. Yuvaraj, P.; Reddy, B. S. R. Tetrahedron Lett. 2014, 55, 806-810. doi:10.1016/j.tetlet.2013.11.116

18. Yao, L.; Yu, X.; Mo, C.; Wu, J. Org. Biomol. Chem. 2012, 10, 9447-9451. doi:10.1039/c2ob26824h

19. Wei, J.; Chen, J.; Xu, J.; Cao, L.; Deng, H.; Sheng, W.; Zhang, H.; Cao, W. J. Fluorine Chem. 2012, 133, 146-154. doi:10.1016/j.jfluchem.2011.09.009

20. Qian, J.; Cao, W.; Zhang, H.; Chen, J.; Zhu, S. J. Fluorine Chem. 2007, 128, 207-210. doi:10.1016/j.jluchem.2006.12.006

21. Lu, L.; Wei, J.; Chen, J.; Zhang, J.; Deng, H.; Shao, M.; Zhang, H.; Cao, W. Tetrahedron 2009, 65, 9152-9156. doi:10.1016/j.tet.2009.09.030 
22. Xu, J.; Wei, J.; Bian, L.; Zhang, J.; Chen, J.; Deng, H.; Wu, X.; Zhang, H.; Cao, W. Chem. Commun. 2011, 47, 3607-3609. doi:10.1039/c0cc05039c

23. Lu, L.; Cao, W.; Chen, J.; Zhang, H.; Zhang, J.; Chen, H.; Wei, J.; Deng, H.; Shao, M. J. Fluorine Chem. 2009, 130, 295-300. doi:10.1016/j.jluchem.2008.11.002

24. Yu, H.; Han, J.; Chen, J.; Deng, H.; Shao, M.; Zhang, H.; Cao, W. Eur. J. Org. Chem. 2012, 3142-3150. doi:10.1002/ejoc.201200180

25. Bian, L.; Xu, J.; Xie, L.; Chen, J.; Deng, H.; Shao, M.; Ding, T.; Zhang, H.; Cao, W. Tetrahedron 2013, 69, 6121-6128. doi:10.1016/j.tet.2013.05.053

26. Wang, Z.; Sun, T.; Chen, J.; Deng, H.; Shao, M.; Zhang, H.; Cao, W. Tetrahedron 2013, 69, 4270-4275. doi:10.1016/j.tet.2013.03.080

27. Ding, Q.; Ye, C.; Pu, S.; Cao, B. Tetrahedron 2014, 70, 409-416. doi:10.1016/j.tet.2013.11.034

28. Chen, Z.; Ding, Q.; Yu, X.; Wu, J. Adv. Synth. Catal. 2009, 351, 1692-1698. doi:10.1002/adsc.200900131

29. Ding, Q.; Chen, Z.; Yu, X.; Peng, Y.; Wu, J. Tetrahedron Lett. 2009, 50, 340-342. doi:10.1016/j.tetlet.2008.11.006

\section{License and Terms}

This is an Open Access article under the terms of the Creative Commons Attribution License

(http://creativecommons.org/licenses/by/2.0), which permits unrestricted use, distribution, and reproduction in any medium, provided the original work is properly cited.

The license is subject to the Beilstein Journal of Organic Chemistry terms and conditions:

(http://www.beilstein-journals.org/bjoc)

The definitive version of this article is the electronic one which can be found at: doi:10.3762/bjoc. 10.238 